\title{
The relationship between cortisol concentrations in late pregnancy and systemic vascular resistance in childhood
}

\author{
P. H. C. Rondó ${ }^{1}$, J. O. Lemos ${ }^{1}$, J. A. Pereira ${ }^{1}$ and J. M.P. Souza ${ }^{2}$ \\ ${ }^{1}$ Nutrition Department and ${ }^{2}$ Statistics and Epidemiology Department, School of Public Health, University of São Paulo, \\ Avenida Dr Arnaldo 715, São Paulo, CEP-01246-904, Brazil
}

Prenatal exposure to glucocorticoids may 'programme' a range of tissue-specific effects in the foetus independently if the exposure is to exogenous glucocorticoids, to active steroids of maternal origin, or to the foetal adrenal products. The consequences for animals and human subjects are consistent with a predominance of cardiometabolic and central nervous system effects ${ }^{(1)}$. The objective of the present study was to assess the relationship between cortisol concentrations in the last trimester of pregnancy and systemic vascular resistance (SVR) in childhood.

The present study is part of a cohort involving 130 pregnant Brazilian women and their children aged 5-8 years ${ }^{(2,3)}$. Maternal cortisol was determined in saliva by an enzyme immunoassay utilising the mean concentration of nine samples of saliva (three in each different day) collected at the same time, early in the morning. SVR was assessed by the HDI/PulseWave CR-2000 Cardiovascular Profiling System $^{\circledR}$ (Hypertension Diagnostics Inc., Eagan, MN, USA). The association between maternal cortisol and SVR in childhood was calculated by multivariate linear regression analysis, controlling for confounding variables.

There was a significant association between maternal cortisol concentrations in saliva and SVR in childhood $(P=0.016)$. The association persisted after controlling for BMI, birth weight, age and gender of the children $(P=0.048)$.

\begin{tabular}{|c|c|c|c|c|c|}
\hline Model SVR & Coefficient & $\mathrm{SE}$ & $95 \% \mathrm{CI}$ & & $P$ \\
\hline \multicolumn{6}{|c|}{ Univariate linear regression } \\
\hline Maternal cortisol & 21.5 & 8.83 & 4.05 & 39.0 & 0.016 \\
\hline Constant & 1946 & 135 & 1678 & 2214 & 0.000 \\
\hline \multicolumn{6}{|c|}{ Multivariate linear regression } \\
\hline Maternal cortisol & 17.90 & 8.96 & 0.17 & 35.6 & 0.048 \\
\hline$B M I$ & -66.0 & 33.7 & -133 & 0.66 & 0.056 \\
\hline Birth weight & -0.08 & 0.14 & -0.35 & 0.19 & 0.549 \\
\hline Age & 7.4 & 13.2 & -18.7 & 33.6 & 0.574 \\
\hline Gender & 56.1 & 131 & -203 & 315 & 0.669 \\
\hline Constant & 2726 & 1176 & 398 & 5054 & 0.022 \\
\hline
\end{tabular}

There are no reported studies that have assessed the association between cortisol concentrations in pregnancy and SVR in childhood. Overall, the data suggest that exposure to excess glucocorticoid in the prenatal period is associated with vascular complications in childhood, predisposing to cardiovascular diseases in later life.

1. Seckl RS (2004) Eur J Endocrinol 151, U49 - U62.

2. Rondo PHC, Ferreira RF, Nogueira F et al. (2003) Eur J Clin Nutr 57, $266-272$

3. Rondo PHC, Lemos JO \& Pereira JA (2008) Clin Sci (Lond) 115, 317 - 326. 